\title{
EDITORIALE
}

\section{COME RENDERE PIÙ CREATIVO IL MARKETING IN UN PERIODO DI CRISI}

\author{
Umberto Collesei ${ }^{\star}$
}

Per fronteggiare la crisi che caratterizza l'economia mondiale, le strategie di mercato più seguite dalle PMI sono due: una che riflette un atteggiamento passivo e un'altra invece un atteggiamento iperattivo. La prima, seguita dalla maggioranza delle imprese, ritiene assai modesto il contributo che il marketing può fornire alla strategia aziendale. Tali imprese, concentrate sulla riduzione dei costi, spostano la loro attenzione dal mercato alla produzione, alla catena di fornitura. Di fatto tendono perciò a impoverire l'offerta e a limitare l'apporto del marketing alla riduzione delle inefficienze distributive e alla razionalizzazione dei processi logistici e di comunicazione.

Sopravvivere a breve sembra essere la loro unica preoccupazione: "non c'è mercato per giustificare nuovi investimenti, non c'è spazio per innovare". A conferma di questa tesi rilevano come si venda quasi solo in promozione, le ricerche di mercato siano troppo costose e troppo lungo il processo di marketing per produrre effetti benefici sulla gestione aziendale. In ogni caso la crisi del commercio, con le numerose chiusure di negozi, sembra una barriera invalicabile per azioni di stimolo della domanda. Secondo questa visione il marketing è chiamato a gestire il quotidiano, a razionalizzare i processi di vendita e comunicazione.

Un altro gruppo di imprese, una minoranza, cerca di superare la crisi dei mercati proponendo in continuazione nuove varianti, nuovi prodotti, spesso frutto di una evoluzione continua, senza sfruttare in pieno le potenzialità offerte dai mercati o dalle nicchie, penetrando marginalmente i nuovi mercati (per l'impresa), così da "salvare il fatturato" con le vendite addizionali.

* Professore ordinario della Facoltà di Economia dell’Università Ca' Foscari Venezia. Mercati e Competitività n. 3, 2010 


\section{U. Collesei}

Entrambe le strategie sono perdenti. La prima si consuma in una guerra di prezzo che non permette alle PMI di acquisire posizioni stabili sul mercato. La seconda se risolve a breve i problemi di sostegno del fatturato non risolve quelli di marginalità, soprattutto si dimostra effimera nel lungo termine perché non permette alle imprese né di difendere la posizione nei vecchi mercati né di acquisire una significativa posizione nei nuovi mercati. Per vincere le sfide originate dalla crisi il contributo del marketing è invece tutt'altro che marginale. Innanzitutto dovrebbe far riflettere l'impresa sulle opportunità che si generano nei diversi mercati, dare rigore metodologico all'analisi, rassicurare sulla possibilità di conseguire risultati di mercato soddisfacenti e durevoli nel tempo. In secondo luogo garantire un continuo monitoraggio dell'evoluzione dei diversi mercati.

Le risposte alla crisi non possono essere solo di breve periodo. Le imprese devono prendere atto che la crisi sta provocando modifiche strutturali dei mercati. Il consumatore del vecchio continente e degli USA non è e non sarà più quello di prima, non solo per il potere di spesa ridotto rispetto a ieri, ma per il diverso approccio al consumo che è stato indotto ad assumere per soddisfare oltre i vecchi anche i nuovi bisogni, tipici di una società opulenta, in parte stimolati dalle nuove tecnologie e dall'invecchiamento della popolazione. Anche la concorrenza non sarà più la stessa, sia per effetto delle innovazioni tecnologiche che sono sfornate a getto continuo dalle imprese innovatrici, sia per effetto della globalizzazione e della dura selezione che ne deriva.

Per avere successo è innanzitutto necessario assumere un'ottica di lungo periodo; in secondo luogo avere una chiara visione strategica della posizione che l'impresa potrà occupare nei diversi mercati. Si dovranno poi verificare le prospettive economico-finanziarie, la capacità di gestire processi e flussi produttivi, distributivi e di comunicazione.

Ovviamente ciò non significa che il marketing non debba fornire il proprio contributo nella gestione a breve dell' attività aziendale, ma solo che se tali apporti sono slegati dalla visione di lungo periodo si corre il pericolo di individuare soluzioni senza prospettive, che addirittura possono mettere a rischio la stessa sopravvivenza dell'impresa. Venendo a "mancare il mercato" cade la stessa ragione d'essere, il presupposto chiave per cui l'impresa opera: perde di valore la marca, la distribuzione non collabora, il prodotto risulta obsoleto.

Dall'altro lato della bilancia vanno però valutati i costi, anche organizzativi, che l'utilizzo di una corretta visione di marketing comporta per le imprese, in particolare per le PMI.

L'evoluzione delle attività e della disciplina ha reso oneroso e complesso gestire una funzione marketing evoluta in tutti gli aspetti della gestione. La consapevolezza di questa implicazione sta facendo passare in seconda linea l'approccio di marketing nell'analisi strategica. Ma esiste un processo low cost di marketing? È possibile realizzarlo? 
La risposta a mio avviso può essere trovata nella separazione tra l'applicazione del metodo di marketing alla formulazione della strategia di impresa e lo sviluppo operativo delle strategie di marketing. La prima, se rigorosa, può dimostrarsi sufficiente se non per individuare le migliori prospettive di mercato per evitare errori decisivi per la sopravvivenza dell'impresa. Il secondo, per produrre gli effetti più significativi, richiede competenze, risorse e tempi lunghi. Ha però il vantaggio di poter essere realizzato gradualmente, via via che cresce l'esperienza di marketing in azienda.

Per l'impresa la soluzione consiste quindi nell'adottare un approccio di marketing strutturato e organizzato per essere implementato nel tempo in base alle esigenze, alle risorse e alle competenze che è in grado di destinare alla realizzazione del processo di marketing.

Se si accetta questa ipotesi di lavoro quale potrebbe essere la metodologia adatta per garantire un efficace approccio di marketing? Essa si articola in sette fasi:

1. analisi dei bisogni;

2. analisi delle risposte delle imprese;

3. verifica delle aree di debolezza;

4. individuazione delle soluzioni percorribili dall'impresa in chiave di prodotto;

5. posizionamento del prodotto e della marca nel mercato;

6. ideazione della logica e delle soluzioni distributive;

7. scelta delle modalità di comunicazione.

1. Analisi dei bisogni

L'analisi dei bisogni è l'attività più difficile da organizzare per una PMI. Richiede, infatti, un continuo monitoraggio della domanda. È la fase dell'ascolto e dell'approfondimento delle insoddisfazioni della domanda, della individuazione di bisogni latenti, della continua evoluzione dei bisogni nel tempo e nei diversi paesi in cui è presente o intende entrare. Essa condiziona pesantemente l'intero approccio di marketing dell'impresa. È invece quella alla quale si destinano di solito poche risorse e competenze.

Per eseguire con successo questa fase è necessario partire da una ricerca di mercato di base che individui correttamente le funzioni chiave e i benefici salienti richiesti al prodotto, che dovrà poi essere continuamente aggiornata attraverso verifiche empiriche e approfondita con indagini ad hoc quando se ne intuiscono possibili modificazioni significative.

2. Analisi delle risposte delle imprese

È questa una fase che è svolta spesso in modo non sistematico dalle PMI. Essa richiede come momento finale una valida classificazione del mercato per individuare i concorrenti più prossimi dell'impresa, l'articolazione del loro marketing mix, la stima dei risultati di mercato da essi con- 
seguiti nei principali mercati di riferimento. La fase si completa con l'analisi delle performance di mercato dell'impresa, in particolare in termini di clienti all'interno dell'universo dei consumatori del mercato o delle nicchie in cui l'impresa è presente e del loro grado di soddisfazione. Questa fase richiede innanzitutto lo sviluppo di un efficace sistema informativo in grado di monitorare costantemente i risultati conseguiti presso la clientela e quindi di integrare i dati interni con quelli esterni che si ottengono dalle indagini sui consumatori.

\section{Verifica delle aree di debolezza}

Il confronto di ciò che chiede/chiederà la domanda e ciò che offrono le imprese che compongono il mercato di riferimento se condotto in stretta sintonia con chi si occupa di progettazione dei prodotti, di produzione e della catena di fornitura dovrebbe consentire di individuare le aree di debolezza delle attuali offerte in termini di tecnologie utilizzate, di soluzioni tecniche, di design, di varietà d'offerta, di prezzi praticati, di impatto ambientale dei prodotti e dei processi, di servizi pre e post vendita forniti dalle imprese concorrenti e dalla nostra impresa.

Si evidenzieranno così delle aree di possibili miglioramenti alla luce anche delle innovazioni tecnologiche che direttamente o indirettamente potranno via via coinvolgere il settore.

4. Individuazione delle soluzioni percorribili dall'impresa in chiave di prodotto

L'upgrading tecnologico del settore e, più specificatamente, delle imprese direttamente concorrenti della nostra e le richieste di nuove funzionalità/prestazioni da parte della domanda (comprese esigenze di miglioramenti in termini di pulizia, conservazione e manutenzione dei prodotti), la ricerca di dare maggiore contenuto esperienziale agli stessi permetterà di individuare alternative di prodotto che dovranno essere verificate in termini di percorribilità di progettazione, approvvigionamento, produzione ed economico-finanziaria, ipotizzando diversi livelli di produzione.

\section{Posizionamento del prodotto nel mercato}

Verificata la percorribilità interna delle diverse soluzioni possibili si dovrà verificarne la marketability, cioè la capacità dell'impresa di sostenere con le proprie risorse e competenze di mercato ed economico-finanziarie le soluzioni di prodotto scelte. In questa fase si dovranno esplicitare i caratteri salienti della marca, dell'impresa e del paese di origine posizionando così la nuova offerta nei mercati di riferimento (quelli nei quali l'impresa già opera o intende entrare).

Da questa analisi l'impresa dovrebbe trarre indicazioni più precise sui volumi che i mercati dovrebbero essere in grado di assorbire. I dati vanno comunque confermati nelle due successive fasi. 
6. Ideazione della logica e delle soluzioni distributive

È anche questa una fase difficile da svolgere da parte delle PMI. In numerosi casi le loro debolezze strutturali riguardano, infatti, la distribuzione e la comunicazione. La prima, in particolare, ne condiziona spesso i risultati di mercato. La presenza, in gran parte indiretta sul mercato distributivo, rende, infatti, difficoltoso imporre la propria strategia di marketing al canale. Anche la possibilità di ottenere una forte collaborazione dai distributori nel lancio e sostegno dei nuovi prodotti è condizionata dalla scarsa capacità economica e organizzativa. Molti sforzi in chiave di prodotto-prezzo da parte dell'impresa produttrice vengono perciò vanificati da un'inefficiente distribuzione.

È questo un punto nodale nello sviluppo del processo di marketing. Le imprese per fare si che le loro strategie di marketing abbiano successo devono investire in organizzazione e gestione del sistema logistico-distributivo, razionalizzando la scelta dei canali e degli intermediari con i quali cercare di sviluppare una collaborazione efficace.

\section{Scelta delle modalità di comunicazione}

L'ultima fase di natura strategica che permette di verificare la percorribilità di mercato dell'offerta che l'impresa intende realizzare riguarda la comunicazione. Va subito precisato che di essa si parla in questa fase, anche se dei vincoli posti dall'immagine si deve tenere conto già nello stadio di sviluppo del prodotto e certamente nella selezione delle soluzioni distributive, tra le quali va tenuto presente, ove possibile, l'e-commerce.

Per le PMI oggi le scelte di comunicazione sono da un lato semplificate dalla presenza del web, dall' altro complicate dalla crescente varietà di media utilizzabili e nello stesso tempo dalle risorse finanziarie di solito scarse che possono destinare a tale attività.

Due le scelte fondamentali alle quali le imprese non possono sottrarsi. La prima, in un'ottica di comunicazione integrata, richiede lo sviluppo di tutte quelle forme di comunicazione minori come i cataloghi, i depliant, la cartellonistica, la carta da lettere, i biglietti da visita, le etichette CD... che devono essere ispirati e riflettere un'immagine coordinata che deriva dallo sviluppo dell'identità di marca; una sua opportuna protezione legale, almeno nei paesi di interesse. La seconda attiene al sito web dell'azienda, che deve divenire il punto di riferimento dell'intera attività di comunicazione e dal quale partire per definire gli altri strumenti di comunicazione di cui l'impresa si può avvalere.

Si completa così la verifica della percorribilità e della convenienza ad attuare una data strategia di mercato. Strategia, ovviamente, che è solo tracciata a grandi linee e che, per essere attuata, ha bisogno di essere tradotta in decisioni operative che richiedono dosi notevolmente più intense di competenze e risorse da destinare all'attività di marketing, in base anche al livello di evoluzione raggiunto dalla cultura di marketing in azienda. 


\section{U. Collesei}

Il processo sinteticamente descritto è certamente alla portata della gran parte delle PMI, non richiedendo elevati livelli di competenze e risorse di marketing ma una chiara e sintetica visione delle opportunità e dei vincoli che il mercato impone alle imprese, in altre parole la semplice adozione dell'approccio di marketing da parte dell'intera impresa.

Una volta messo a punto appare però essenziale che l'impresa si attrezzi per monitorarlo e aggiornarlo costantemente. È questo un momento cruciale per lo sviluppo del marketing in azienda, che richiede una formalizzazione in un piano, almeno in un documento sintetico, che ne evidenzi i punti salienti e che ne testimoni la condivisione da parte dell'intera impresa e della direzione in particolare. Ad esso si dovrà fare riferimento nello sviluppo dei piani e dei progetti operativi che certamente saranno condizionati tra l'altro dal grado di evoluzione che il marketing operativo avrà raggiunto in impresa.

Così inteso, nei suoi aspetti fondamentali, il marketing si presenta nella sua essenza: un marketing delle idee, un modo di progettare l'attività dell'impresa partendo dal mercato, più attento a ciò che chiede la domanda $\mathrm{e}$ ciò che offre l'offerta.

In un mondo sempre più complesso, difficile da decifrare, lungi dal semplificare la realtà, l'approccio di marketing propone oggi la forza delle idee semplici, dell'attenzione e della comunicazione continue in cui l'ascolto e la collaborazione del cliente sono fondamentali per individuare le soluzioni che con il contributo della creatività aziendale avranno successo nel mercato. Un' analisi che verifica la percorribilità della proposta innanzitutto in chiave di mercato e poi in termini di capacità di interna gestione, in grado di adattarsi alle mutevoli condizioni ambientali e dei mercati in senso sia spaziale sia temporale.

Per avere successo nel mercato l'impresa deve trovare un'idea forte da concretare in una proposta significativa, soprattutto altamente differenziata dalla concorrenza e allo stesso tempo in grado di soddisfare al meglio le esigenze salienti della domanda.

E quindi nel rapporto tra marketing, progettazione, produzione, approvvigionamenti che va stimolata la riflessione strategica prima e la pianificazione operativa poi.

Per chi presidia l'attività di marketing in azienda si richiede, oggi rispetto a ieri, oltre ad una valida conoscenza delle problematiche aziendali e delle tecniche specifiche di marketing una maggiore conoscenza dei mercati, una metodologia rigorosa dell' ascolto e di comunicazione, un approccio creativo nella continua interazione con i mercati e con la tecnologia. 